\title{
Growing Local Likelihood Network: Emergence of Communities
}

\author{
S. Chen and M. Small (a) \\ School of Mathematics and Statistics, The University of Western Australia, Crawley, WA, Australia, 6009
}

PACS 89.75.Da - Systems obeying scaling laws

PACS 89.75. Fb - Structures and organization in complex systems

\begin{abstract}
In many real situations, networks grow only via local interactions. New nodes are added to the growing network with only information pertaining to a small subset of existing nodes. Multilevel marketing, social networks, and diseases models can all be depicted as growing networks based on local distance information. In these examples, all nodes whose distance from a chosen center is less than $d$ form a subgraph. We thence grow networks with information only from these subgraphs. Moreover, we use a likelihood-based method, where at each step we modify the networks in the way that will make the graph closer to the expected degree distribution. Combining local information and the likelihood method, we grow networks that exhibit novel features. We discover that the likelihood method, at certain parameter ranges, can generate networks with highly modulated communities, even when global information is not available. Communities and clusters are abundant in real-life networks, and the method proposed here provides a natural mechanism for the emergence of communities in scale-free networks. In addition, the algorithmic implementation of network growth via local information is substantially faster than global methods and allows for the exploration of much larger networks.
\end{abstract}

Introduction. - Many systems in the physical and biological realms exhibit behaviours governed by local rather than global interaction. Growth of such systems is often governed by local knowledge - when a new node is added to the network, it is not provided with perfect global information. Examples abound in different fields $[1,2]$. Social relationship, computers under virus attack, and insects affected by infectious diseases all form networks based on local evolving rules.

After Barabási and Albert's investigation into scale-free networks [3], there has been a huge amount of work devoted to the growth, properties and applications of these networks. The mechanism of the growth, the hub, and the size effect has been discussed relating to the scale-free properties [4-7]. In contrast to growing networks from a global view, local-world scale-free networks has the advantage that it is, in many situations, closer to reality [8]. Moreover, most of these networks prove to be scale-free [9].

However, despite the inherent localisation of some net-

\footnotetext{
(a) E-mail: michael.small@uwa.edu .au
}

works, most growth algorithms operate with global structural information of the network. In fact, the difficulty in modelling local behaviour is how we should define "local" when encountering the restriction to local information in particular a localisation method that maintains the intuitive appeal of Barabási's preferential attachment is still lacking. Current methods have necessarily introduced additional mechanistic parameters, such as generating subgraph by "graph affinity" [10], randomly selecting $m$ existing nodes $[8,11]$, or choosing nodes according to clustering coefficient [12]. Distance information, however, has rarely been explored. (Here we clarify that although there are many spatial networks with "distance, such as transportation network, our "distance" is in graphical sense rather than true "physical" distance.) Aldridge [13] showed that networks based on subgraphs constructed with distance information exhibit similar properties to that of global information. The main difference lies in that local ones have a fatter tail in the degree distribution.

In addition, regarding how to grow the network, preferential attachment provides the obvious and intuitive answer $[3,14]$. However, it is not clear that it is the correct one [15]. Conversely, the recent introduction 
of a likelihood-based approach gives a natural and well defined way of choosing between candidate nodes. The idea of likelihood is proposed and elucidated in [16] and [17]. By referring to likelihood, we mean the probability of a graph being selected $P\left(G_{N}\right)$ among all the graphs $G_{N}$ of $N$ nodes. It is computed based on an underlying probability distribution (power-law distribution) and the degree sequence of $G_{N}$. Here we explore the problem with local information: at each step, we select a center node and nodes within distance $d$ from the center form a subgraph. Then we introduce a mechanism to add nodes or edges within the subgraph as long as the operation increases $P\left(G_{N}\right)$, the likelihood of the new graph where likelihoods are only computed with the available local information. Moreover, many real world networks exhibit clusters and communities, but it is unclear how a simple growth algorithm can explain this. Furuya and Yakubo [18] discusses the community structure on local-evolving scale-free network, but only via the BA method. In this paper, we will show the novel properties that the networks generated via this likelihood method exhibit, especially community modularity. We compare this likelihood-based local growth method to a version of preferential attachment, driven only by local information.

Different Approaches Towards Local Distance Information. - For graph $G$ with $N$ nodes, we want to restrict our knowledge to part of the network. Since we base our understanding on distance information, a center or focal node is chosen to define the locale, and the local restriction lies in that only nodes within some distance of that focal node are assumed to be "known". The value of that distance, defines the extent of knowledge of the local vs. global information.

Here are the three methods we propose to generate and examine such subgraphs:

(a) Random Focus Subgraph At each step, one node is randomly chosen from all the existing nodes to be the new focal node, and all the nodes within distance $d$ from the focus are selected as the new subgraph.

(b) Low-Degree Focus Subgraph Same as (a), except for selecting the focal node according to the power law $p_{k} \propto k^{-\gamma}$, making low-degree nodes (the peripheral of a network) more likely to be chosen as the focus.

(c) Wandering Focus Subgraph At each step, one node is randomly selected from nodes in the previous subgraph as the new focal node, and all the nodes within distance $d$ from the focus are selected as the new subgraph.

\section{Grow Networks via Local Information. -}

Likelihood Calculation. For a graph $G_{N}$ with $N$ nodes, let $n_{k}$ denote the number of nodes with $k$ links in $G_{N}$.
Then we plot out $n_{i}$ versus $i$ to form the histogram of degree distribution. A scale-free network is usually defined to be a graph for which the degree distribution obeys the power law:

$$
p_{k} \propto k^{-\gamma} .
$$

As stated in [19], this definition refers to the asymptotic distribution. The following procedure proposed in [19] generates networks that are more "likely" to be scalefree.

The likelihood of $G_{N}$ conforming to degree distribution $p_{k}$ is

$$
P\left(G_{N}\right)=N ! \prod_{k=1}^{N} \frac{p_{k}^{n_{k}}}{n_{k} !}
$$

So, at each step, one node is added or one link is added between an existing node and the new node. We compute the marginal payoff $Q$ s of these operations.

For adding a new node: see (3)

with $\tilde{p}=1-\sum_{k=1}^{N} p_{k}$.

For adding an edge from the last added node to a node of degree $k$ (suppose that the last added node is of degree $j)$ : see $(4)$

These $Q$ s represent the effect of the operation: if $Q$ is larger than 1, then the current step makes the graph more scale-free, and vice versa. Given all these $Q_{\text {node }} s$ and $Q_{e d g e-j} s$, we can determine which operations will make the graph better fit the target degree distribution. A move may then be selected from among these candidate operations probabilistically with probablility proportional to the $Q_{\mathrm{s}}$.

Alternatively, one can generate a graph by deterministically selecting the biggest $\mathrm{Q}$ at each step (optimal?): the graph would always be close to the expected degree distribution.

Here we propose a parameteric approach to allow us to choose between the "optimal" to somewhat "random" approach:

(i) Compute $Q_{\text {node }}$ and $Q_{\text {edge-j }}$ at each step;

(ii) Rearrange the $Q \mathrm{~s}$ from highest to lowest $\left(Q_{1}^{\prime}, Q_{2}^{\prime} \ldots\right)$ and normalize the sum of $Q$ to 1 . Choose a monotonically decreasing function as bias function;

(iii) Select a step according to the area of each $Q^{\prime}$ under bias function.

Hence the steeper the bias function, the more likely the higher $Q \mathrm{~s}$ are chosen. We describe the steep bias function as "optimal" ("more scale-free?"), and the flat bias function as "random". In our simulation, we used exponential decreasing function $e^{-\xi}$ and adjust the parameter $\xi$. 


$$
Q_{\text {node }}(k)=\frac{P\left(G_{N+1}\right)}{(N+1) \tilde{p} P\left(G_{N}\right)}=\frac{(N+1) ! \prod_{k=1}^{N} \frac{p_{k} n_{k}^{\prime}}{n_{k}^{\prime} !}}{(N+1) \tilde{p} N ! \prod_{k=1}^{N} \frac{p_{k} n_{k}}{n_{k} !}}=\left\{\begin{array}{cc}
\frac{p_{2}}{n_{2}+1} \frac{1}{\tilde{\tilde{p}}} & k=1 \\
\frac{p_{1}}{n_{1}+1} \frac{n_{k}}{p_{k}} \frac{p_{k+1}}{n_{k+1}+1} \tilde{\tilde{p}} & k>1
\end{array}\right.
$$

$$
Q_{\text {edge }-j}(k)=\frac{P\left(\tilde{G}_{N,(j+1)}\right)}{P\left(G_{N}\right)}=\left\{\begin{array}{cc}
\frac{n_{j-1}}{p_{j-1}} \frac{p_{j+1}}{n_{j+1}+1} & k=j-1 \\
\frac{p_{j+1}^{2}}{\left(n_{j+1}+1\right)\left(n_{j+1}+2\right)} \frac{n_{j}\left(n_{j}-1\right)}{p_{j}^{2}} & k=j \\
\frac{n_{j}}{p_{j}} \frac{p_{j+2}}{n_{j}+2+1} & k=j+1 \\
\frac{n_{j}}{p_{j}} \frac{p_{j+1}}{n_{j+1}+1} \frac{n_{k}}{p_{k}} \frac{p_{k+1}}{n_{k+1}+1} & |k-j|>1
\end{array}\right.
$$

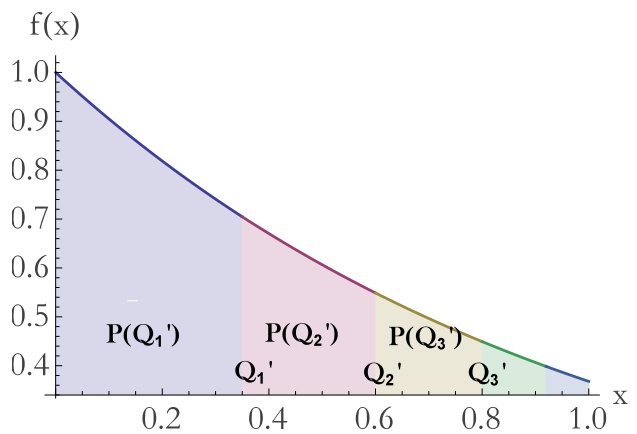

Fig. 1: Steps in selecting steps for growing networks via likelihood method. The $Q^{\prime}$ s are marginal payoffs of each steps, and $P\left(Q^{\prime}\right)$ s, the probability of selecting $Q^{\prime}$ s, are proportional to the area under the bias function. When the bias function $f(x)$ is steep, larger $Q$ s are more likely to be selected.

Likelihood Method with Local Information. Previous work on the likelihood method has only explored network properties via a global approach. We find that, when combined with local distance information, this method provides new insight into both community structure and other graph properties. To explore this we propose two different assumption in our restriction of information:

(i) Absolute Blindness Compute $Q_{\text {node }} s$ and $Q_{\text {edge-j }} s$ only on the subgraphs generated by one of the three methods and select moves from the local results.

(ii) Partial Knowledge Compute $Q_{\text {node }} S$ and $Q_{\text {edge-j }} s$ on all of the existing nodes, but execute the move only within the subgraph.

Preferential Attachment with Local Information. The local approach can also be combined with the BarabásiAlbert preferential attachment method. We assume that at each step, one only knows about the local information. At each step, one node is added and $m$ edges are linked between the new node and $m$ existing nodes with linking probability proportional to the degree of the nodes.
Hence our network generation could be divided into two parts: (1) generating subgraphs; (2) growing networks. We will mainly focus on how the likelihood method differs with preferential attachment and how the two assumptions have their impact on the network properties. A schematic is provided in figure 2 .

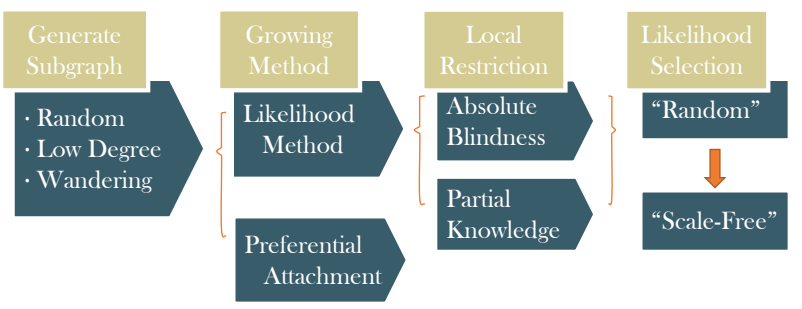

Fig. 2: Schematic of the process: generate subgraphs and grow networks via different approaches.

Simulation Results. - We compare different approaches for generating subgraphs and growing networks. In all these simulations, the Wandering Focus method does not differ significantly from the Random Focus and LowDegree Focus. The Wandering Focus Subgraph will "circle around" the whole graph and give everywhere an asymptotic scale-free degree distribution, just as the random approach does. This is true only when the $d$, the subgraph size, is not too small and we have verified that for $d$ larger than 2, their network properties (community modularity, global clustering coefficient, and graph diameter etc.) differ by less than $10 \%$. In addition, since low-degree nodes are most plentiful in scale-free networks, the Low-Degree Focus Subgraph gives almost the same results compared to the Random Focus. Hence, in what follows we summarise our results are based on the Random Focus method for generating subgraphs, unless stated otherwise (Fig. 8). And the $\gamma$ of the underlying degree distribution is set as 2.5, except those stated in Fig. 5.

Community Structure Emerges. The likelihood method has its strongest advantage in that the community structure emerges automatically when we adjust the parameters. Here we assume Absolute Blindness, and gen- 

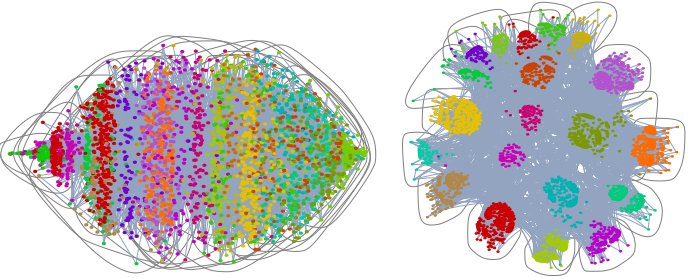

(a) $\xi=10, \operatorname{Mod}=0.419$

(b) $\xi=30, \operatorname{Mod}=$ 0.400

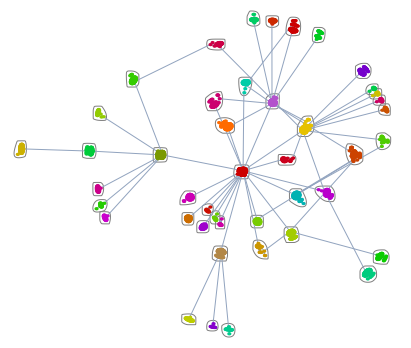

(c) $\xi=50, \operatorname{Mod}=0.946$

(d) $\xi=70, \operatorname{Mod}=0.891$

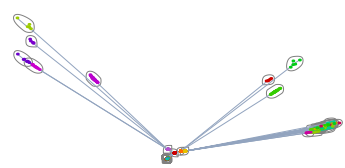

(e) $\xi=90, \operatorname{Mod}=0.863$

Fig. 3: Community Plots of networks with different parameter $\mathrm{k}$ via the likelihood method. Five graphs differ in the parameter $\xi$, from 10 to 90 . The distance information restriction is the same: $d=5$ with a RandomFocus, and we assume Absolute Blindness. From (a) to (e), we adjust the selection method from random to optimal, inducing a low and progressively increasing preference for higher $Q$ - steps that would give more scalefree results. It turns out that highly modulated community structure emerges for intermediate parameter $\xi$. erate graphs using likelihood method with fixed distance information $(d=5$ and a Random Focus Subgraph). There are 3000 nodes in each graph.

As can be seen in Fig. 3, community structure emerges when we adjust $\xi$ from 10 to 90 - from random to optimal. When $\xi=50$, there is a clear community structure with the highest community modularity. For larger $\xi$, there is still a community structure, but with fewer modules and less proportion of interconnection between different communities among all the links.

Different parameters of the networks shows a clear transition over the interval from $\xi=30$ to $\xi=40$, as shown in Fig. 4. A series of changes break out in the structure of the network. The community modularity rises significantly. This clear structure might appear as a result of transition from a rather "random" approach to a more "optimal" one. More communities emerge from the process of adding edges when the likelihood to connect to the nodes with highest degree decreases, meaning that new nodes are more likely to be interconnected in the lo-

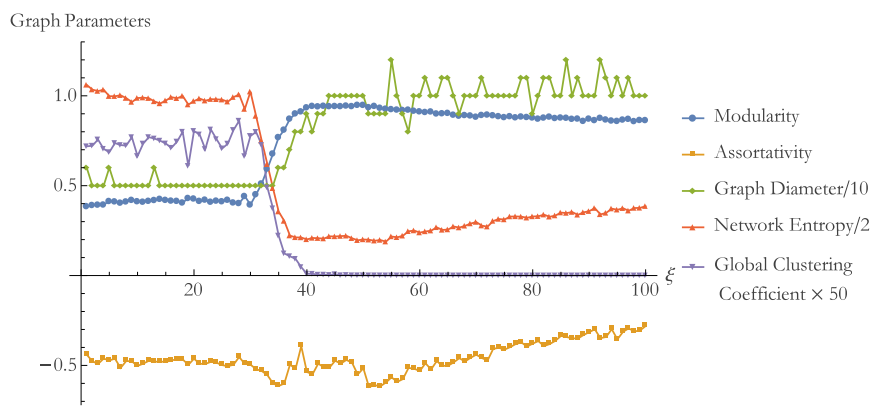

Fig. 4: Network parameters of networks generated with different $\xi$. The graph diameter and network are scaled to better show these different parameters. The exponential decay rate $\xi$ ranges from 1 to 100 , and a clear transition emerges around $\xi=35$ where community modularity, graph diameter and network entropy shows a clear transition. The modularity rises significantly, from 0.4 to near 1 , indicating highly structured communities.

cal subgraphs. This apparent structure also reduces the network entropy. Moreover, the global clustering coefficient drops sharply, resulting from less closed triplets in the network. The network diameter, on the other hand, rises significantly, since more wandering distance within communities are required from one end to reach the other end of the network. Strikingly, these new features emerge sharply from the smoothly adjusted parameter.

This transition is not a coincidence of a speicial underlying degree distribution. In Fig. 5, we present the relationship between $\gamma$, the power-law exponent of the underlying degree distribution, and the transition of graph modularity. Similar to Fig. 4, networks are generated with the parameter $\xi$ varying from 1 to 50 , but with different $\gamma$. The transition from "random" to "optimal" exhibits a shift: larger $\gamma$ makes the transition happen earlier. It can be explained by the fact that the higher $\gamma$ means steeper digree distribution, thus higher probability to grow "optimally".

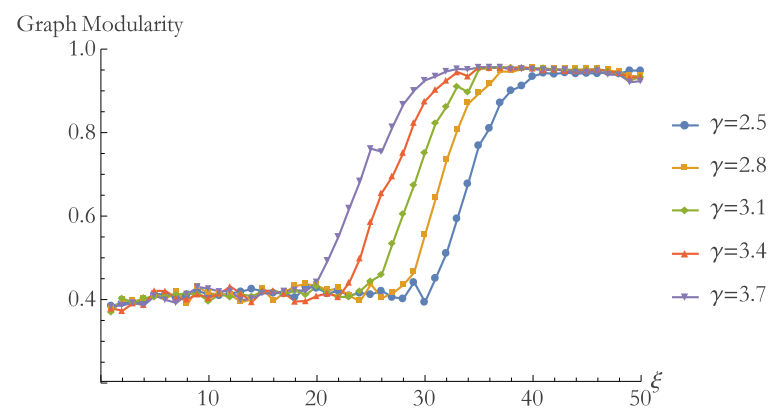

Fig. 5: Community Modularity for networks generated with different $k$ and underlying degree distribution. The transition from "random" to "optimal" is observed in all $\gamma$, and the transition interval is shifted to smaller $\xi$ when $\gamma$ is increased.

Local vs Global. When we fix the parameter $\xi=50$, we can explore how the restriction in distance information 
affects the properties of the networks. Here we still use the Random Focus subgraph, and the likelihood method with the Absolute Blindness assumption.

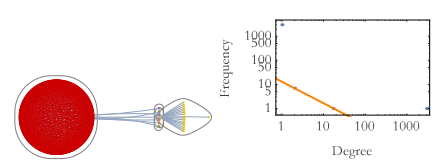

(a) $\quad d=1, \operatorname{Mod}=0.013$

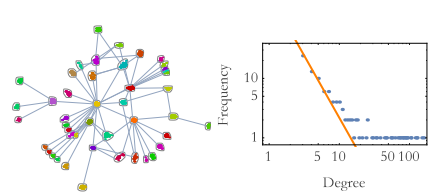

(c) $\quad d=5, \operatorname{Mod}=0.946$

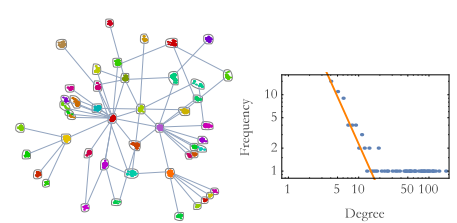

(e) Global $\operatorname{Mod}=0.946$

Fig. 6: Community Plots of Networks with different restriction of information (from $d=1$ to $d=5$ ). The $\xi$ is fixed to 50 , and the Random Focus subgraph is used with the Absolute Blindness assumption. We show 3000 nodes per network and observe that (i) at very small $d$ (very limited information), we do not not generate scale-free networks. (ii) as $d$ increases, it converges to the case of Global Information, meaning that the Absolute Blindness gives almost the same results.

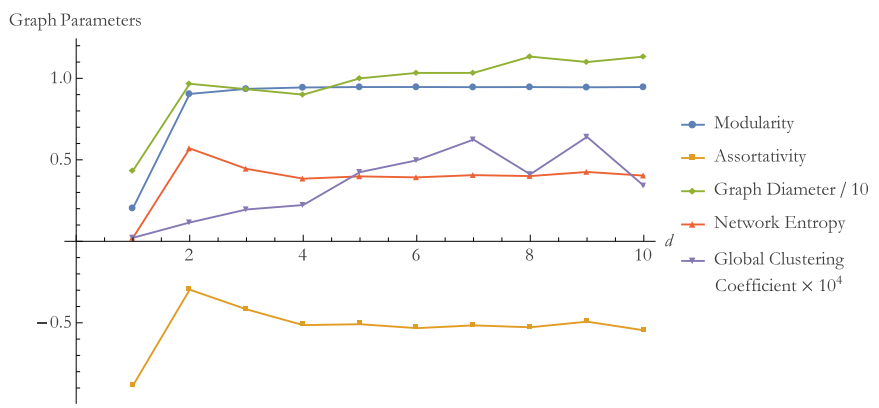

Fig. 7: Network parameters of networks generated with different subgraph sizes. The graph diameter and network are scaled to better show different parameters. The subgraph size ranges from $d=1$ to $d=10$. The parameter $\xi$ is fixed as 50. Aside from $d=1$, parameters of the graphs are similar. That implies that in most cases the limited information would generate networks resembling those via global information. In addition, global clustering coefficients are small, so we present the average results to avoid sampling noise.

As shown in Fig. 6, too limited global knowledge would result in a non-scale-free network. Additional knowledge of global degree distribution would compensate for this deficiency (by computing $Q_{\mathrm{s}}$ on the whole network). See Fig.
7. This could be explained by the fact that when $d=1$, the subgraphs are extremely small, and our algorithm would give a biased choice at each step, most likely towards the higher-degree node. Moreover, when the neighborhood is enlarged, we can see that the local approach converges to the global one - exactly as we would expect.

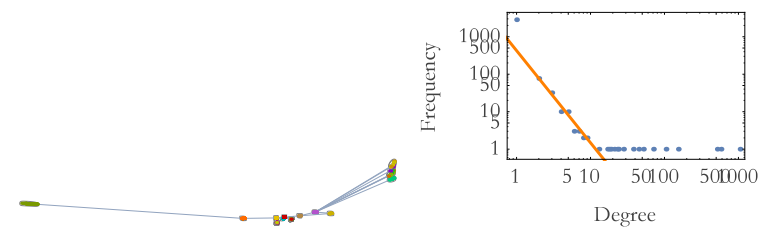

Fig. 8: Wandering Focus compensates for the failure to generate scale-free network when information is too limited. The subgraph size is $d=1$. Subgraphs are selected by Wandering Focus. Its community modularity is 0.778 , much higher than the Random Focus 0.013. The $\gamma$ is 2.46 .

Another way to compensate for this failure is to apply Wandering Focus. The wandering focal provides a better "view" for the network to grow. (Fig. 8) Rather than frequently attaching to a super-node, it provides a possibility to grow the network in one direction further, thus avoiding the expansion of the super-node in the center.

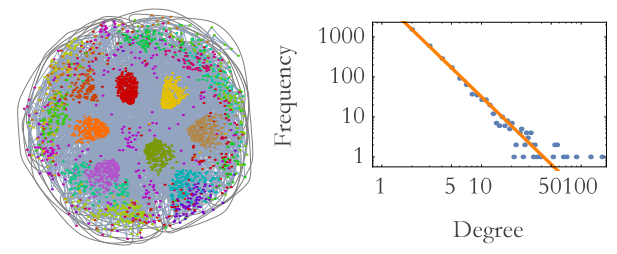

Fig. 9: Network generated via BA preferential attachement, with subgraph distance 5 . The modularity is 0.532 . Compare it with Fig. 6(c), which is generated by local likelihood method with $d=5$. The likelihood method generates networks more modulated than BA method.

Comparison with BA method. The local BA preferential attachment model generates graphs without community structure, while our local method gives highly modulated communities. We compare the BA method $(m=2, d=5)$ and likelihood method (Absolutely Blindness, $d=5, \xi=50$ ). Results are depicted in Fig. 9.

In addition to community structure, the degree distribution is different too. This could be viewed as a consequence of the highly modulated communities. In the likelihood method, the degrees tend to be stratified - more nodes with same degree in each community than those via BA method. This stratification might have its counterpart in the real world where interconnections among communities are much higher than between communities. This, in turn, leads to strong degree assortativity. (Assortativity for BA: -0.06 , for Likelihood: -0.56) 
Computational Efficiency. - For these algorithms, we compare their computational efficiency. All the calculations are done in Mathematica, and scaled so that the time for global approach is 1 .

\begin{tabular}{cccc}
\hline Distance & Partial Knowl. & Absolute Blind. & Global \\
\hline $\mathrm{d}=1$ & 4.30 & 7.15 & \\
\cline { 1 - 3 } $\mathrm{d}=2$ & 1.58 & 0.35 & \\
\cline { 1 - 3 } $\mathrm{d}=3$ & 0.98 & 0.30 & \\
\hline $\mathrm{d}=4$ & 0.96 & 0.54 & \\
\hline $\mathrm{d}=5$ & 0.97 & 0.71 &
\end{tabular}

Table 1: The time scale of different approaches, compared to the global method. We set the global method as the standard time 1 and compare the Mixed Approach with $\xi=50$. We compute all the time of computing $Q \mathrm{~s}$ and adding nodes and edges. The Absolute Blindness assumption is faster than Partial Knowledge when the distance information is limited, because less time is spent on re-choosing subgraphs.

When our algorithm is run under the Absolute Blindness assumption, the efficiency is the highest. See Table 1. The Partial Knowledge assumption is slower, mainly because it is constantly regenerating subgraphs. As the distance increases, it also becomes slower, because more information needs to be processed at each step.

Conclusion. - In this paper, we examine different approaches to growing networks based on local information and different algorithms to realise these approaches. These are all based on the likelihood method, aiming at growing networks with certain rules extracted from the underlying degree distribution that we set beforehand. Our premise, which we have demonstrated in this work, is that growing networks with only local information naturally leads to the emergence of community structure.

Growing networks via likelihood method can generate highly scale-free networks, since, if we take the most optimal probability density function we require that at each step our modification should make the degree distribution closer to the target [19]. If we restrict our attention and information to only some subgraphs, we shall get scale-free networks, but with a prevalent community structure. This observation should help explain the origin and evolution of highly-modulated networks in the real world.

While varying the parameters in the proposed network growth algorithms, we discover a transition beyond which graphs exhibit a highly modulated community structure. This transition is sharp and reveals a "boundary" between the "random" scale-free network and "optimal"??? scale-free network. The possible explanation would be that local information gives only local clusters - more links between members within the same community than between the members of different community. In more "optimal" situations, results are more like the real global scheme, where less community structure can be observed. With well-adjusted parameters, new nodes would not only connect to the focal node of each subgraph, but also to the nodes within the subgraphs, building the modular community structure. In addition, compared to standard preferential attachment based methods, our approach might provide a more natural explanation to highly modulated communities, which has the potential application in future research of networks with communities.

$$
* * *
$$

MS is funded by the Australian Research Council via a Future Fellowship (FT110100896) and Discovery Project (DP140100203). Mathematica implementations of the algorithms described in this paper are available from the first author.

\section{REFERENCES}

[1] Liljeros F., Edling C. R., Amaral L. A. N., Stanley H. E. and Åberg Y., Nature, 411 (2001) 907.

[2] Small M., Walker D. M. and Tse C. K., Phys. Rev. Lett., 99 (2007) 188702.

[3] BArabÁsi A.-L. and Albert R., science, 286 (1999) 509.

[4] Boguná M., Pastor-Satorras R. and Vespignani A., The European Physical Journal B-Condensed Matter and Complex Systems, 38 (2004) 205.

[5] Colizza V., Flammini A., Serrano M. A. and VespigNANI A., Nature physics, 2 (2006) 110.

[6] Newman M. E., Contemporary physics, 46 (2005) 323.

[7] Baglietto G. and Albano E. V., Physical Review E, 78 (2008) 021125.

[8] Li X. and Chen G., Physica A: Statistical Mechanics and its Applications, 328 (2003) 274.

[9] Newman M., Networks: an introduction (Oxford University Press) 2010.

[10] Gómez-Gardeñes J. and Moreno Y., Phys. Rev. E, 69 (2004) 037103

[11] Sun S., Liu Z., Chen Z. and Yuan Z., Physica A: Statistical Mechanics and its Applications, 373 (2007) 851

[12] Náther P., Markošová M. and Rudolf B., Physica A: Statistical Mechanics and its Applications, 388 (2009) 5036.

[13] ALDRIDGE C., Scale-free networks using local information for preferential linking in proc. of MODSIM 2005 International Congress on Modelling and Simulation, Modelling and Simulation Society of Australia and New Zealand, December 2005 pp. 1196-1202.

[14] Callaway D. S., Hopcroft J. E., Kleinberg J. M., Newman M. E. J. and Strogatz S. H., Phys. Rev. E, 64 (2001) 041902.

[15] Liu J., Abbass H. A., Zhong W. and Green D. G., Artificial life, 17 (2011) 263.

[16] Judd K., Small M. and Stemler T., EPL (Europhysics Letters), 103 (2013) 58004.

[17] Small M., Hou L. and Zhang L., National Science Review, (2014). 
[18] Furuya S. and Yakubo K., Physica A: Statistical Mechanics and its Applications, 389 (2010) 5878.

[19] Small M., Li Y., Stemler T. and Judd K., Phys. Rev. E, 91 (2015) 042801. 